\title{
The prevalence of ADH1B and OPRM1 predisposing alleles for alcohol consumption are increased in the Hungarian psoriasis population
}

\author{
Zita Szentkereszty-Kovácsa ${ }^{a}$ Szilvia Fiatal ${ }^{b}$, Eszter Janka ${ }^{a}$, Krisztina Herszényic, Péter Hollóc, \\ Andrea Szegedia,d , Éva Remenyik ${ }^{a}$, Dániel Törőcsik ${ }^{a}$, e
}

aDepartment of Dermatology, Faculty of Medicine, University of Debrecen, Debrecen, Hungary

${ }^{b}$ Department of Preventive Medicine, Faculty of Public Health, University of Debrecen, Debrecen, Hungary

'Department of Dermatology, Venereology and Dermatooncology, Semmelweis University, Budapest, Hungary

${ }^{d}$ Division of Dermatological Allergology, Faculty of Medicine, University of Debrecen, Debrecen, Hungary

\section{INTRODUCTION}

Psoriasis vulgaris (PsV) is a common systemic inflammatory disease with numerous genetic and environmental factors in the background. Although alcohol consumption is a key environmental factor affecting in great the symptoms and life of PsV patients, its genetic association with PsV remains to be elucidated.

\section{AIMS}

The objective of our study was to investigate the association of psoriasis with several established alcohol consumption and dependence related gene variants.

\section{RESULTS}

Differences between the psoriatic and Hungarian General population remained significant only for one SNP (rs1229984) after multiple test correction. Comparing the allele frequency distribution of SNP rs1229984 in the gene coding alcohol dehydrogenase 1B (ADH1B) significantly higher prevalence of effect allele ' $C$ ' was found in the psoriatic population compared to the HG population $(94.46 \%$ vs. $92.04 \%, p<0.001$, respectively). Significant differences were observed between study groups in the association analysis according to the additive (OR=1.58, $95 \% \mathrm{Cl}=1.23-2.03, \mathrm{p}<0.001)$ and the recessive model (OR=1.58, 95\% $\mathrm{Cl}=1.22-2.04$ $\mathrm{p}=0.001)$ but not the dominant model $(\mathrm{OR}=4.29,95 \% \mathrm{Cl}=0.55-33.08, \mathrm{p}=0.163)$

Table 1. Effect allele frequency distribution in rs1229984

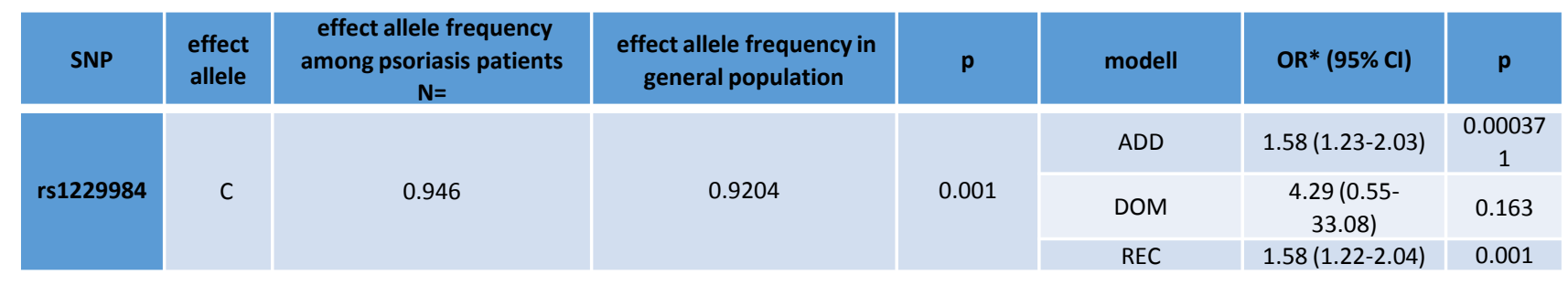

By comparing the psoriasis subgroups several strata of psoriatic patients were defined, such as familial aggregation vs. sporadic case, early-onset of disease $(\leq 40$ years) vs. late-onset ( $\geq 40$ years) and PASI score $(\leq 10, \geq 10)$. Significant results were found only in case of one SNP rs1799971 ( $\mu$-opioid receptor gene, OPRM1) when familial cases were compared to sporadic cases. The effect allele $G$ increased the risk of familial psoriasis by two fold compared to sporadic cases both in additive and in dominant models (ORadditive=1.99, ORdominant=2.01; $p<0.001$ ).

Table 2. Association of rs 1799971 with psoriasis vulgaris analyzed by subphenotype stratification

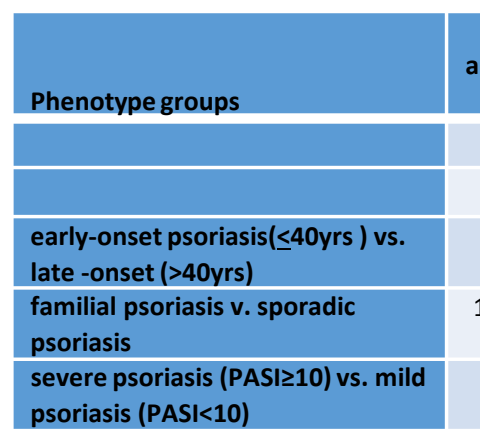

\begin{tabular}{|c|c|}
\hline $\begin{array}{c}\text { additive (GG } \\
\text { vs. AA) }\end{array}$ & p-val \\
\hline OR(95\%) & \\
\hline \begin{tabular}{c|c}
$0.66(0.44-$ \\
$1.006)$
\end{tabular} & 0.0537 \\
\hline $1.993(1.36-$ & \\
$2.91)$ & 0.0003 \\
\hline $0.97(0.65-$ & 0.8997 \\
$1.45)$ & \\
\hline
\end{tabular}

\begin{tabular}{|c|c|c|c|c|}
\hline p-value & $\begin{array}{l}\text { recessive (GG } \\
\text { vs. GA+AA) }\end{array}$ & p-value & $\begin{array}{c}\text { dominant } \\
(G G+G A \text { ss. } \\
\text { AA) }\end{array}$ & $\mathrm{p}$-value \\
\hline & $\mathrm{OR}(95 \%)$ & & OR(95\%) & \\
\hline 0.0537 & $1.17(0.17-7.85)$ & 0.8711 & $\begin{array}{c}0.62(0.39- \\
0.97)\end{array}$ & 0.035 \\
\hline 0.0003 & $4.53(0.77-26.4)$ & 0.092 & $\begin{array}{c}2.01(1.35- \\
3.01)\end{array}$ & 0.0006 \\
\hline 0.8997 & $0.79(0.13-4.85)$ & 0.8076 & $\begin{array}{c}1.05(0.68- \\
1.59)\end{array}$ & 0.8291 \\
\hline
\end{tabular}

\section{MATERIALS AND METHODS}

A population based case-control study including 3743 subjects (776 PsV cases and 2967 controls from the general Hungarian population) was conducted. Genotyping of 23 single nucleotide polymorphisms (SNPs) were determined using Mass Array platform with iPLEX Gold Chemistry (Sequenom). Differences in genotype and allele distributions were investigated. Multiple logistic regression analyses were also implemented.

\section{Statistical analyses}

The data were analyzed using STATA 12.0 Statistical software (StataCorp LP, College Station, TX, USA). The Mann-Whitney $U$ and $\chi 2$ tests were used to compare the mean age and sex distribution of the two study groups. The existence of HardyWeinberg equilibrium (HWE) and significant differences in the allele and genotype frequencies between the two populations were examined with the $\chi 2$ test. After adjusting for multiple comparisons (Bonferroni correction), a p-threshold of 0.002 was applied; otherwise the threshold for significance was 0.05
DISCUSSION

In this study we evaluated the relationship between 23 SNPs related to increased alcohol intake and dependence in a psoriasis group from Hungary. We found that the frequency of the genetic variant rs1229984 (ADH1B gene) increased in the whole psoriasis group, while the prevalence of genetic variant rs1799971 (OPRM1 gene) showed higher prevalence in the familial form of psoriasis patients. Importantly, the psoriasis risk related to these variants increased further in the subgroup of psoriatic patients with history of early onset and familial aggregation.

ADH1B is a key enzyme in the metabolism of ethanol to acetaldehyde and subsequent oxidation to acetate. The allele $\mathrm{G}$ with a detected increased prevalence in the psoriasis population leads to an increased enzyme activity, thus to decreased levels of the harmful acetaldehyde following the alcohol ingestion.

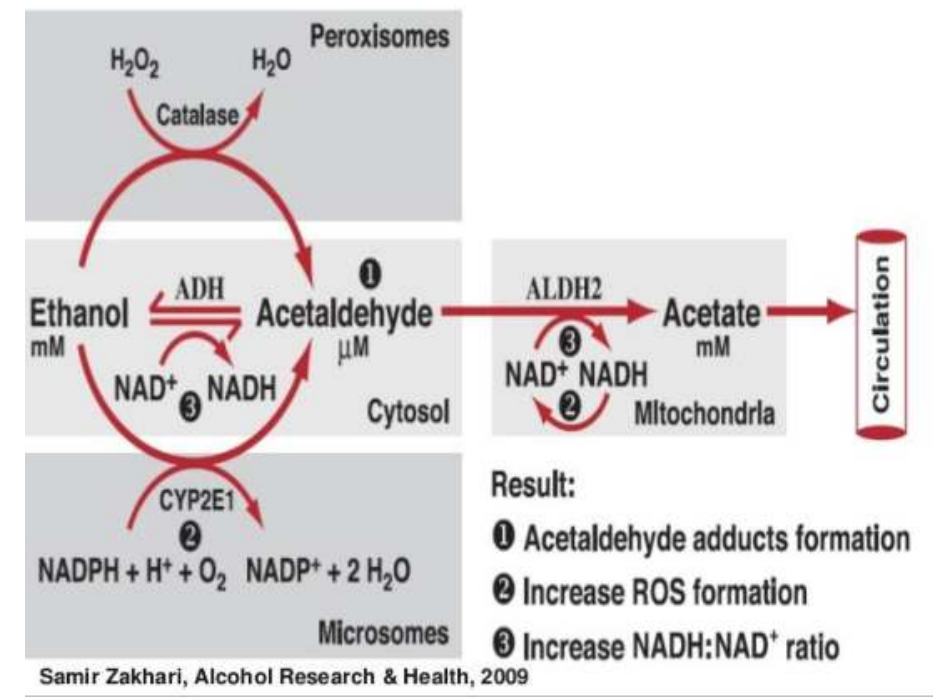

OPRM1 encodes the $\mu$-opioid receptor, which upon activation by its ligands, such as opioids and analgesic agents like beta-endorphin, modulates the dopamine system [1]. It is implicated in complex behavior patterns such as alcohol dependence in Caucasian, native American tribes as well as in a study group of Asian descends [2-5] in alcohol dependence associated impulsivity [6] just as in a reduced response to rewarding stimuli $[7,8]$. Out of the alleles, $118 \mathrm{G}$ has the major susceptive effect. In individuals carrying 118G stimulation, sedation, and positive mood levels after alcohol intake were significantly higher than in controls [8].

In conclusion, our data suggests that genetically defined high-risk individuals for alcohol consumption are more common among psoriasis patients than in the general population in Hungary, which highlights the importance to integrate the genetic determinants of health behavior into our current thinking of psoriasis diagnosis and treatment. These findings therefore also set the basis and the need for novel implications, besides focusing only on the role of non-genetic factors, in planning and delivering effective preventive interventions targeted for psoriatic patients.

References

Johnson SW, North RA: Opioids excite dopamine neurons by hyperpolarization of local interneurons. The Journal of neuroscience : the official journal of the Society for Neuroscience 12: 483-488, 1992.

Kranzler HR Gelernter J O'Malley S. Hernandez-Avila CA Kaufman D: Association of alcohol or other drug depend deerch 22: 1359-1362 1998.

Chen D Liu L Xiao Y, Peng Y Yang C, Wang Z: Ethnic-specific meta-analyses of association between the OPRM1 A118G polymorphism and alcohol dependence among Asians and Caucasians. Drug and alcohol (20) 1-6, 2012 .

Anlers CL, Gizer IR: Evidence for a genetic component for substance dependence in Native Americans. The American journal of psychiatry 170: 154-164, 2013

Koller G, Zill P, Rujescu D, Ridinger M, Pogarell O, Fehr C, et al.: Possible association between OPRM 1 genetic variance at the 118 locus and alcohol dependence in a large treatment sample: relationship to alcoho dependence symptoms. Alcoholism, clinical and experimental research 36: 1230-1236, 2012.

Pfeifer P Sariyar M, Eggermann T, Zerres K, Vernaleken I, Tuscher O et al: Alcohol Consumption in Healthy OPRM1 G Allele Carriers and Its Association with Impulsive Behavior. Alcohol and alcoholism (Oxford, Oxfordshire) 50: 379-384, 2015.

Lee MR, Gallen CL, Zhang X, Hodgkinson CA, Goldman D, Stein EA, et al.: Functional polymorphism of the mu-opioid rector Ray LA, Hutchison KE: A polymorphism of the mu-opioid reptor of alcohol in humans. Alcoholism, clinical and experimental research 28: 1789-1795, 2004. 